\title{
Identificação de Enterobacteriaceae da microbiota intestinal de frangos de corte submetidos a dieta com nitrofuranos
}

\author{
Enterobacteriaceae identification of the broiler intestinal \\ microbiota submitted to nitrofurans diet
}

\author{
Carla Inês Soares Praxedes, ${ }^{*}$ Nathália Oliveira Cavalcanti Zúniga, ${ }^{* * *}$ \\ Paula Aparecida Martins Borges Bastos, ${ }^{* * *}$ Robson Maia Franco, ${ }^{* *}$ Sérgio Borges Mano**
}

\begin{abstract}
Resumo
Objetivou-se neste trabalho identificar Enterobacteriaceae da microbiota intestinal de frangos de corte que foram submetidos a quatro tratamentos diferentes com nitrofuranos, do $15^{\circ}$ ao $23^{\circ}$ dia de vida. Foram coletadas, por via cloacal, amostras fecais através de suabes estéreis de 52 aves da linhagem Cobb, em seguida transportados em meio Cary-blair para isolamento e identificação dos gêneros e espécies através das provas bacteriológicas convencionais e através dos sistemas Bactray I e II. Os gêneros e espécies isolados nos tratamentos com nitrofuranos e no controle foram: Citrobacter spp., Escherichia coli, Escherichia fergusonii, Escherichia Hermannii, Enterobacter aerogenes, Enterobacter aminigenus, Enterobacter cloacae, Enterobacter sakazakii, Hafnia alvei, Klebsiella oxytoca, Klebsiella ozaenae, Klebsiella pneumoniae, Morganella morganii, Proteus spp e Pseudomonas luteola. De todos os tratamentos, o maior percentual de Escherichia coli isoladas ocorreu no grupo controle, sendo este também o que apresentou menor diversidade de micro-organismos, com apenas dois gêneros identificados. O uso de antimicrobianos na ração nos demais tratamentos pode ser uma explicação para o desequilíbrio da microbiota normal do intestino, resultando em um aumento populacional de outras enterobacteriaceas.
\end{abstract}

Palavras-chave: antimicrobianos, enterobactérias, isolamento.

\begin{abstract}
The objective of this study was to identify Enterobacteriaceae of the broiler intestinal microbiota submitted to four different treatments involving nitrofurans, from the 15th to the 23th day of life. Fecal samples, from the chickens' sewers, were collected through sterilized swabs from 52 chickens of the Cobb strain, afterwards taken into Cary-blair method to isolation, and identification of genus and species through the conventional bacteriological tests and Bactray I/II systems. The genera and species isolated in nitrofurans treatments and in the control were: Citrobacter spp., Escherichia coli, Escherichia fergusonii, Escherichia Hermannii, Enterobacter aerogenes, Enterobacter aminigenus, Enterobacter cloacae, Enterobacter sakazakii, Hafnia alvei, Klebsiella oxytoca, Klebsiella ozaenae, Klebsiella pneumoniae, Morganella morganii, Proteus spp e Pseudomonas luteola. Of all treatments, the highest percentage of isolated Escherichia coli occurred in the control group, being the latter the one which presented the lowest microorganism diversity - only two genera were identified. The use of antibiotics in the feed on other treatments can be an explanation the imbalance of the common tract microbiota, resulting in a population growth of other Enterobacteriaceae.
\end{abstract}

Keywords: antimicrobial, enterobacteria, isolation.

\section{Introdução}

Durante o desenvolvimento embrionário do pinto não há qualquer microbiota natural intestinal, que se forma a partir da ingestão de micro-organismos durante o processo de nascimento, aumentando nas primeiras semanas de vida até se tornar uma população predominantemente de bactérias anaeróbicas. Alguns gêneros e espécies bacterianos que colonizam inicialmente o trato intestinal, geralmente persistem ao longo de toda a vida das aves, passando a compor a microbiota intestinal residente
(Furlan et al., 2004; Palermo-Neto et al., 2005). Apajalahti et al. (2004), utilizando técnicas que caracterizam o DNA microbiano extraídos de colônias do trato gastrointestinal, mostraram que $90 \%$ das bactérias encontradas são de espécies desconhecidas.

Neste contexto, o estudo da microbiota instestinal tem sido motivo de diversas investigações científicas, principalmente pelo relacionamento que se estabelece entre o hospedeiro e os organismos que são albergados (Silva et al., 2004). Estudos recentes apontam para o aumento considerável na proporção

\footnotetext{
* Programa de Pós-graduação em Medicina Veterinária (Doutorado) - Higiene Veterinária e Processamento Tecnológico de Produtos de Origem Animal Faculdade de Veterinária - Universidade Federal Fluminense. E-mail: cispraxedes@hotmail.com. Av. Dário Vieira Borges, 235, Lia Márcia, Bom Jesus do Itabapoana - RJ. CEP: 38360-000. Tel.: (22)3833-9850.

** Docente do Departamento de Tecnologia dos Alimentos da Faculdade de Veterinária da Universidade Federal Fluminense.

*** Médica Veterinária do Departamento de Microbiologia de Alimentos do câmpus Bom Jesus do Itabapoana do Instituto Federal Fluminense.

**** Programa de Pós-graduação em Ciência de Alimentos (Doutorado) - Instituto de Química - Centro de Ciências Matemáticas e da Natureza - Universidade Federal do Rio de Janeiro.
} 
de micro-organismos resistentes e multirresistentes, agravandose com maior rapidez comparando com décadas anteriores (Hernández et al. 2004; Palermo-Neto et al., 2005).

Seres humanos e animais podem se contaminar por bactérias patogênicas de difícil controle terapêutico, a partir de alimentos e ambientes contaminados (Costa et al., 2009), advindos principalmente do uso do promotor de crescimento na ração (Pessanha e Gontijo Filho, 2001). Os nitrofuranos pela sua eficácia e baixo custo foram muito utilizados como profilático em coccidioses e salmoneloses, terapêutico nas infecções por E. coli, Salmonella spp., Mycoplasma gallisepticum e enterites inespecíficas e como aditivo zootécnico antibacteriano e antiprotozoário em aves e suínos (Magalhães et al., 1985; Mottier et al., 2005; Barbosa et al., 2007; Price, 2009).

Desde 1990, está proibido na União Europeia o uso destes compostos na criação de animais de produção (União Europeia, 1990) e no Brasil através da Instrução Normativa nº 9 de 27 de Junho de 2003 do Ministério da Agricultura (Brasil, 2003a). Entretanto, têm sido utilizados de forma ilegal, constatados em produtos de origem animal no Sul e Sudeste da Ásia, Brasil e Portugal (Kennedy, 2003) e mais recentemente Bangladesh, Índia e Sri Lanka (Rasff, 2009).

Neste sentido, objetivou-se no presente trabalho avaliar a ocorrência de enterobacteriaceas da microbiota intestinal de frangos de corte submetidos à dieta com nitrofuranos.

\section{Material e métodos}

O presente trabalho foi realizado no Instituto Federal Fluminense, através de um acordo tripartite interinstitucional feito em 2008, denominado "Acordo de Cooperação Acadêmica e Intercâmbio Técnico, Científico e Cultural” entre o Instituto de Química da Universidade Federal do Rio de Janeiro (UFRJ), a Faculdade de Veterinária da Universidade Federal Fluminense (UFF) e o Instituto Federal Fluminense (IFF) campus Bom Jesus, naquele ano denominado Colégio Agrícola Ildefonso Bastos Borges, vinculado à Universidade Federal Fluminense (UFF).

O experimento de campo foi realizado em galpão experimental do campus Bom Jesus, sendo adquiridos 275 pintos de um dia (machos e fêmeas) da linhagem Cobb, provenientes do incubatório da empresa Globoaves da cidade de Formiga, Minas Gerais.

Os animais foram separados em cinco grupos de 55 (clinicamente sadios) e criados em condições experimentais, feita da forma mais próxima possível de uma criação comercial, mimetizando o manejo de um avicultor. No manejo de criação as aves receberam durante os primeiros14 dias de vida ração de fase inicial (milho, soja e núcleo de fase inicial) e água potável à vontade provida em bebedouros pendulares durante todo o experimento. No décimo quinto iniciou-se o tratamento com as rações medicadas contendo dosagens terapêuticas de acordo com Zuidema et al. (2004) e McCracken et al. (2005), por sete dias consecutivos.

Para a ração medicada no tratamento um contendo $185 \mathrm{mg} / \mathrm{kg}$ de furazolidona (FZD), foram homogeneizados $3,7 \mathrm{~g}$ de furazolidona da SIGMA-ALDRICH (St. Louis, MO, USA) em 1,0 kg de ração de milho e soja adicionada de núcleo em saco plástico fechado. Após a homogeneização foram adicionados mais $19 \mathrm{~kg}$ de ração, completando $20 \mathrm{~kg}$ de alimento medicado, sendo feita a homogeneização do total. Para a ração medicada do tratamento dois contendo $202 \mathrm{mg} / \mathrm{kg}$ de furaltadona (FTD), foram homogeneizados 4,04 g de furaltadona da SIGMA-ALDRICH (St. Louis, MO, USA) conforme descrito para a furazolidona. Para a ração medicada do tratamento três contendo $300 \mathrm{mg} / \mathrm{kg}$ de nitrofurantoína, foram homogeneizados $6,0 \mathrm{~g}$ de nitrofurantoína (NFT) da SIGMA-ALDRICH (St. Louis, MO, USA) da mesma forma como descrito para a furazolidona. Para a ração medicada do tratamento quatro contendo $300 \mathrm{mg} / \mathrm{kg}$ de nitrofurazona, foram homogeneizados 6,0g de nitrofurazona (NFZ) da SIGMAALDRICH (St. Louis, MO, USA) em conformidade com o descrito para a furazolidona. No tratamento cinco, designado ao grupo controle, os animais continuaram recebendo a ração de fase inicial, porém sem a adição de antimicrobiano.

Após os sete dias de tratamento, restituiu-se a criação normal, retornando a alimentação com ração comercial de fase de crescimento, que foi administrada até os 42 dias de vida, quando houve a substituição por ração de fase de finalização, administrada até os 51 dias de vida.

Procederam-se seis abates de grupos de animais de todos os tratamentos entre o $23^{\circ}$ dia de vida e o $51^{\circ}$ dia, com objetivo de coletar amostras para futuro estudo de metabolização e distribuição de resíduos de nitrofuranos por pesquisadores da Universidade Federal do Rio de Janeiro (Zúniga, 2010). No $51^{\circ}$ dia de vida, no momento anterior ao abate, foi realizada a coleta de amostra através de suabe cloacal do último grupo de aves (52 aves) para posterior identificação bacteriana.

As 52 amostras fecais (suabes) foram distribuídas da seguinte forma: nove suabes do tratamento um contendo furazolidona, 11 suabes do tratamento dois contendo furaltadona, 11 suabes do tratamento três contendo nitrofurantoína, 12 suabes do tratamento quatro contendo nitrofurazona e nove suabes do tratamento cinco, denominado controle. Após, os suabes foram acondicionados em tubos de ensaio esterilizados contendo o meio Cary-blair (meio de transporte) da marca STERILE e enviados para o Laboratório de Microbiologia do campus Bom Jesus. Cada ave (frango) foi representada por um suabe (amostra fecal).

As amostras mantidas em meio Cary-blair foram analisadas para detecção de Enterobacteriaceae de acordo com a metodologia descrita pela Instrução Normativa SDA $\mathrm{N}^{\circ}$ 62 de 26 de agosto de 2003, do Ministério da Agricultura, Pecuária e Abastecimento (Brasil, 2003b) para as fases de pré-enriquecimento, enriquecimento seletivo, isolamento e identificação bioquímica de triagem.

Todas as colônias foram submetidas a análises bioquímicas específicas através do sistema Bactray I e II para identificação de Enterobacteriaceae.

Os resultados foram expressos em percentuais de ocorrência dos micro-organismos encontrados em cada tratamento e no grupo controle.

\section{Resultados e discussão}

$\mathrm{Na}$ Tabela 1 estão apresentadas todas as enterobactérias identificadas no sexto e último abate de frangos $\left(51^{\circ}\right.$ dia de criação), separadas de acordo com o tratamento dos nitrofuranos e o grupo controle. 
Tabela 1: Microrganismos isolados a partir de suabes cloacais de frangos de corte com 51 dias de idade submetidos à dieta com nitrofuranos

\begin{tabular}{|c|c|c|c|c|c|}
\hline & FZD & FTD & NFT & NFZ & CONTOLE \\
\hline Citrobacter spp. & & & $3 \%$ & & \\
\hline Escherichia coli & $72 \%$ & $47 \%$ & $57 \%$ & $33 \%$ & $76 \%$ \\
\hline Escherichia fergusoni & $4 \%$ & & & $6 \%$ & \\
\hline Escherichia hermannii & & & $3 \%$ & & \\
\hline Enterobacter aerogenes & $4 \%$ & & & & \\
\hline Enterobacter aminigenus & & & & $3 \%$ & \\
\hline Enterobacter cloacae & & & $7 \%$ & $3 \%$ & \\
\hline Enterobacter sakazakii & & $3 \%$ & $3 \%$ & $3 \%$ & \\
\hline Hafnia alvei & $4 \%$ & $6 \%$ & & $3 \%$ & \\
\hline Klebsiella oxytoca & $4 \%$ & $3 \%$ & $7 \%$ & & \\
\hline Klebsiella ozaenae & $12 \%$ & $22 \%$ & & $13 \%$ & $20 \%$ \\
\hline Klebsiella pneumoniae & & $10 \%$ & $3 \%$ & $23 \%$ & $4 \%$ \\
\hline Morganella morganii & & & $7 \%$ & & \\
\hline Proteus spp. & & & $10 \%$ & & \\
\hline Pseudomonas luteola & & $9 \%$ & & $13 \%$ & \\
\hline
\end{tabular}

FZD = Furazolidona; FTD = Furaltadona; NFT = Nitrofurantoína; NFZ = Nitrofurazona; Controle $=$ Sem medicação

O gênero Salmonella não foi identificado em nenhuma das 52 amostras pesquisadas, o que pode ser resultado de uma criação que envolveu as boas práticas agropecuárias, pois apesar de ser uma criação experimental, nesta procurou-se mimetizar a ambiência de uma granja comercial, ou então devido à utilização dos quimioterápicos (nitrofuranos) na ração, evitando a colonização de Salmonella spp., dificultando sua detecção pelos métodos tradicionais (Tellez et al., 2001).

Coutinho et al. (2009) também não identificaram Salmonella spp., Listeria spp., Campylobacter spp. ao analisarem suabes cloacais de avestruzes, porém, foram isoladas E. coli $(95 \%)$, Pseudomonas aeruginosa (28,3\%), Klebsiella spp. (8,33\%), Proteus spp. e Enterobacter spp. (1,66\%).

Observou-se que o percentual de Escherichia coli nos grupos com os tratamentos contendo furaltadona, nitrofurantoína e nitrofurazona $(47 \%, 57 \%$ e $33 \%$, respectivamente) foram inferiores ao encontrado no grupo tratado com furazolidona (72\%) e no controle (76\%).

Observou-se também que nas aves do grupo controle ocorreu menor diversidade na microbiota intestinal, com a presença de apenas dois gêneros, dentre esses, três espécies (Escherichia coli, Klebsiella ozaenae e Klebsiella pneumoniae). Avaliando gêneros por grupo, a maior diversidade foi encontrada no tratamento com a nitrofurantoína, onde foram isolados seis gêneros diferentes, no tratamento com a nitrofurazona e a furaltadona cinco gêneros diferentes e com a furazolidona quatro gêneros diferentes.

Uma possível explicação para a maior diversidade de gêneros nos grupos tratados com os derivados dos nitrofuranos pode estar no desequilíbrio causado pela oferta do quimioterápico sobre a microbiota intestinal normal do frango. Reynolds et al. (1997) apontaram para o fato de que o uso de antimicrobianos pode exacerbar a população de algumas enterobacteriaceae devido ao desequilíbrio da microbiota normal do intestino. No grupo controle, que ocorreu a menor diversidade de gêneros, houve maior percentual de Escherichia coli comparado a todos os outros grupos. Alto percentual de Escherichia coli também foi encontrado de forma semelhante, 88\% por Sáenz et al. (2001) e $57 \%$ por Silva et al. (2004).

$O$ alto percentual de $E$. coli encontrado no grupo controle se deve provavelmente à colonização normal do trato gastrintestinal das aves, pois inúmeras espécies de bactérias iniciam a colonização imediatamente após o nascimento das aves e aumenta durante as primeiras semanas de vida, até se tornar uma população predominantemente de bactérias anaeróbicas (Furlan et al., 2004).

No grupo tratado com furazolidona também houve aumento no percentual de Escherichia coli isoladas (72\%), que provavelmente se justifica pelo menor efeito da furazolidona em relação a este micro-organismo, visto que Manning et al. (1994) ao introduzirem Salmonella enteritidis resistentes a nitrofurazona em aves de corte com sete dias de idade, alimentadas com ração contendo nitrofurazona, verificaram redução significativa da concentração de ácidos graxos voláteis no ceco, que influenciam a susceptibilidade frente a alguns patógenos entéricos.

Para Ito et al. (2000), quando manipulamos diferentes tipos de ingredientes ou aditivos alimentares, fenômenos ainda pouco compreendidos podem estar ocorrendo com a microbiota intestinal das aves, e de forma inusitada, ocorrendo perda de seus efeitos benéficos ou exacerbação de seus efeitos nocivos.

Segundo Lancini (1994), a microbiota bacteriana do trato entérico das aves tem um efeito benéfico, atuando no processo digestório, e de acordo com Ito et al. (2000), de uma forma indireta, a presença da microbiota intestinal equilibrada, particularmente no ceco, contribui com a exclusão e/ou competição com bactérias patogênicas como E.coli, Clostridium spp. e Salmonella spp. Muitas das espécies identificadas no presente trabalho foram detectadas no intestino e/ou ceco de aves, como é o caso de Citrobacter, Enterobacter, Proteus, Escherichia coli, Klebsiella (Furlan et al., 2004; Silva et al., 2004; Palermo-Neto et al., 2005)

As aves podem ser portadoras de diversos micro-organismos, patogênicos ou não, e esses possivelmente podem ser transmitidos a outras aves (Silva et al., 2004), podendo apresentar-se isoladas ou associadas. São também responsáveis por surtos epidêmicos ou casos isolados de perturbação intestinal em humanos através do consumo de alimentos contaminados (Costa e Hofer, 1972).

\section{Conclusões}

Apesar de procurar mimetizar a ambiência de uma granja comercial, este procedimento foi uma criação experimental, sendo conduzida dentro das boas práticas agropecuárias. Foram isoladas diversas espécies bacterianas, em especial a Escherichia coli, que se apresentou em todas as aves, sob efeito dos quimioterápicos ou não, principalmente com percentual elevado no grupo controle $(76 \%)$ e no grupo tratado com furazolidona (72\%).

A detecção do grau de susceptibilidade dessas bactérias aos nitrofuranos pode ser uma fonte de informação importante para explicar a diversidade bacteriana entre os distintos tratamentos com os nitrofuranos e o controle desse quimioterápico, existindo 
poucos dados na literatura científica. A preocupação com casos de resistência bacteriana e até mesmo transferência de genes de resistência constituem uma intranquilidade para com a saúde coletiva, e sua disseminação via cadeia alimentar pode diminuir as opções de tratamento nas infecções causadas por micro-

\section{Referências}

APAJALAHTI, J.; KETTUNEN, A.; GRAHAM, H. Characteristics of the gastrointestinal microbial communities, with special reference to the chicken. World's Poultry Science Journal, n. 60, p. 223-232, 2004.

BARBOSA, J.; MOURA, S.; BARBOSA, R.; RAMOS, F.; SILVEIRA, M. Determination of nitrofurans in animal feeds by liquid chromatography-UV photodiode array detection and liquid chromatography -ionspray tandem mass spectrometry. Analytica Chimica Acta, v. 586, p. 359-365, 2007.

BRASIL. Ministério da Agricultura, Pecuária e Abastecimento. Instrução Normativa $n^{\circ} 9$ de 27 de Junho de 2003a. Proíbe a fabricação, a manipulação, o fracionamento, a comercialização, a importação e o uso dos principais ativos cloranfenicol e nitrofuranos e os produtos que contenham estes princípios ativos, para uso veterinário e suscetível de emprego na alimentação de todos os animais e insetos. Diário Oficial da República Federativa do Brasil, Brasília, DF, p. 4, 30 de junho de 2003.

BRASIL. Ministério da Agricultura, Pecuária e Abastecimento. Instrução Normativa SDA $n^{\circ} 62$ de 26 de agosto de 2003b. Métodos Analíticos Oficiais para Análises Microbiológicas para Controle de Produtos de Origem Animal e Água. Diário Oficial da República Federativa do Brasil, Brasília, DF, 265 p., 18 de setembro de 2003. COSTA, R.A.; HOFER, E. Isolamento e identificação de enterobactérias (Apostila). Rio de Janeiro: Instituto Oswaldo Cruz, 120 p., 1972. COSTA, M.M.; MALBONI, F.; WEBER, S.S.; FERRONATO, A.I.; SCHRANK, I.S.; VARGAS, A.P.C. Patotipos de Escherichia coli na suinocultura e suas implicações ambientais e na resistência aos antimicrobianos. Arquivo Instituto de Biologia, São Paulo, v. 76, n. 3, p. $509-516,2009$.

COUTINHO, C.E.R.; FRANCO, R.M.; MAGALHÃES, $H_{.}$; AQUINO, M.H.C. Investigação de Salmonella spp., Listeria spp., Campylobacter spp. e Pseudomonas spp. no trato intestinal de avestruzes (Struthio camelus) criados no estado do Rio de Janeiro. Revista Brasileira de Ciência Veterinária, v. 16, n. 3, p. 129-132, 2009.

FURLAN, R.L.; MACARI, M.; LUQUETTI, B.C. Como avaliar o efeito de uso de probióticos, prebióticos e flora de exclusão competitiva. In: $5^{\circ}$ Simpósio Técnico de Incubação, Matrizes de Corte e Nutrição. Balneário Camboriú, SC, 2004.

HERNÁNDEZ, F.; MADRID, J.; GARCIA, V. Influence of two plant extracts on broilers performance, digestibility, and digestive organ size. Poultry Science, v. 83, p. 169-174, 2004.

ITO, N.M.K.; MIYAJI, C.I.; LIMA, E.A.; OKABAYASHI. Enfermidades do Sistema Digestório e Anexos. In: BERCHIERI JÚNIOR, A; MACARI, M. Doenças das Aves. Campinas: FACTA, 2000, p. 47-60.

KENNEDY, G. Nitrofuranos em Avicultura. IV Simpósio Brasil Sul de Avicultura. Chapecó, SC, 2003.

LANCINI, J.B. Fatores exógenos na função gastrointestinal. In: Fisiologia da Digestão e Absorção das Aves. Fundação Apinco, 1994. p. 99-126.

MAGALHÃES, H.M.; BOELTER, R.; SIVA, A.R. Elementos de Farmacologia Veterinária. 3. ed. Porto Alegre: Ed. Sulina, 1985. $199 \mathrm{p}$.

McCRACKEN, R.J.; VAN RHIJN, J.A.; KENNEDY, D.G. The occurrence of nitrofuran metabolites in the tissues of chickens exposed to very low dietary concentrations of the nitrofurans. Food Additives and Contaminants, v. 22, n. 6, p. 567-572, 2005. organismos patogênicos ou comensais humanos. Mediante tal ponderação, a realização do perfil de resistência dos microorganismos identificados no presente trabalho é de extrema importância, sendo objeto de outra pesquisa em andamento.

MANNING, J.G.; HARGIS, B.M.; HINTON, A.; CORRIER, D.E.; DeLOACH, J.R.; CREGER, C.R. Effect of selected antibiotics and anticoccidials on Salmonella enteritidis cecal colonization and organ invasion in Leghorn chicks. Avian Diseases, v. 38, n. 2, p. 256-261, 1994.

MOTTIER, P.; KHONG, S.P.; GREMAUD, E.; RICHOZ, J.; DELATOUR, T.; GOLDMANN, T.; GUY, P.A. Quantitative determination of four nitrofuran metabolites in meat by isotope dilution liquid chromatography-electrospray ionization-tandem mass spectrometry. Journal of Chromatography A, v. 1067, p. 85-91, 2005.

PALERMO-NETO, J.; SPINOSA, H.S.; GÓRNIAK, S.L. Farmacologia aplicada à avicultura: Boas Práticas no Manejo de Medicamentos. Editora Roca Ltda, 2005.

PESSANHA, R.P.; GONTIJO FILHO, P.P. Uso de antimicrobianos como promotores de crescimento e resistência em isolados de Escherichia coli e de Enterobacteriaceae lactose-negativa da microbiota fecal de frangos de corte. Arquivo Brasileiro de Medicina Veterinária e Zootecnia, v. 53, n. 1, 2001.

PRICE, P. Residues meat Instruction: General residues background information. 2009. Disponível em: <http://www.dardni.gov.uk/vphumoc-chapter-05-residues.pdf>. Acesso em 14 de julho de 2010.

RASFF. The Rapid Alert System for Food and Feed. Annual Report 2009. Disponível em: <ec.europa.eu/food/food/.../report2009_ en.pdf>. Acesso em: 21 de junho de 2011.

REYNOLDS, D.J.; DAVIES, R.H.; RICHARDS, M.E.; WRAY,C. Evaluation of combined antibiotic and competitive exclusion treatment in broiler breeder flocks infected whith Salmonella enterica serovar enteritidis. Avian Pathology, v. 26, n. 1, p. 83-95, 1997.

SÁENZ, Y.; ZARAZAGA, M.; BRIÑAS, L.; LANTERO, M.; RUIZLARREA, F.; TORRES, C. Antibiotic resistance in Escherichia coli isolates obtained from animals, foods and humans in Spain. International Journal of Antimicrobial Agents, v. 18, p. 353-358, 2001.

SILVA, G.M.; SILVA, C.M.F.;BRUNO, S.F.; ABREU, D.L.C Identificação de Enterobacteriaceae da microbiota intestinal de aves de postura (Gallus gallus Linnaeus, 1758) da linhagem Lohmann S.L.S. Revista Brasileira de Ciência Veterinária, v. 11, n. 3, p. 153-155, 2004.

TELLEZ, G.; PETRONE, V.M.; ESCORCIA, M.; MORISHITA, T.I.; COBB, C.W.; VILLASEÑOR, L. Evaluation of avian-specific probiotic and Salmonella enteritidis, Salmonella typhimurium, Salmonella Heidelberg-Specific antibodies on cecal colonization and organ invasion of Salmonella enteritidis in broilers. Journal Food Protection, v. 64, n. 3, p. 287-291, 2001.

UNIÃO EUROPEIA Council Regulation 2377/90/EC of 26 June 1990, laying down a Community procedure for the establishment of maximum residue limits of veterinary medicinal products in foodstuffs of animal origin. Official Journal of the European Union L224, 18/08/1990.

ZUIDEMA, T.; VAN RHIJN, J.A.; SCHAT, B.; MULDER, P.P.J; BOLCK, Y.J.C.; HOOGENBOOM, L.A.P. e KENNEDY, D.G. Metabolism and depletion of furazolidone and furaltadone in broilers. In: Proceedings of the Euroresidue $V$ Conference, Ginkel L.A. (van), Bergwerff A.A., editors. Noordwijkerhout, The Netherlands, 12 may 2004. Bilthoven: RIVM, p. 996-1001, 2004.

ZÚNIGA, N.O.C. Desenvolvimento de criação experimental de frango de corte para viabilização de estudo de metabolização de nitrofuranos. 2010. 211 f. Dissertação (Mestrado) - Instituto de Química - Universidade Federal do Rio de Janeiro, Rio de Janeiro, 2010. 